\title{
SÍNDROME DE BURNOUT: CONFRONTO ENTRE O CONHECIMENTO MÉDICO E A REALIDADE DAS FICHAS MÉDICAS
}

\author{
Jaqueline Brito Vidal Batista \\ Mary Sandra Carlotto" \\ Antônio Souto Coutinho \\ Lia Giraldo da Silva Augusto ${ }^{\Phi}$
}

\begin{abstract}
RESUMO. A partir da ampliação de sua prática profissional, incluindo, além do ensino, atividades de cuidado para com o aluno e a missão de garantir a articulação entre a escola e a comunidade, o professor se tornou mais suscetível a desenvolver a Síndrome de Burnout, doença ocupacional contemplada pelo Ministério da Saúde desde 1999. Um dos responsáveis pelo seu diagnóstico institucional é a perícia médica. Dada a importância do diagnóstico correto da Síndrome de Burnout, esse estudo teve como objetivo investigar, através da aplicação de questionário e da consulta a fichas médicas, o conhecimento da perícia médica da Junta Médica Municipal da cidade de João Pessoa, PB, a respeito dessa síndrome. Os resultados apontaram o desconhecimento da legislação e da Síndrome de Burnout e a necessidade de uma intervenção de políticas públicas que proporcionem um maior preparo dos médicos peritos e uma melhor assistência à saúde mental do professor.
\end{abstract}

Palavras-chave: Síndrome de Burnout; saúde mental; saúde do professor.

\section{SYNDROME OF BURNOUT: A CONFRONTATION BETWEEN MEDICAL KNOWLEDGE AND MEDICAL RECORDS REALITY}

\begin{abstract}
From the expansion of his practice, including, in addition to teaching, activities of student care and the mission to ensure the link between the school and community, the teacher became more susceptible to developing the Syndrome of Burnout, occupational disease covered by the Ministry of Health since 1999. One of the institutional responsibility for their diagnosis is the medical expertise. Due the importance of correct Syndrome of Burnout diagnosis, this study aimed to investigate, through the implementation of the questionnaire and medical records consultation, the knowledge of medical expertise from the Medical Board of the João Pessoa city, PB, about this syndrome. The results pointed to the ignorance about legislation and Burnout of Syndrome and to the need for public policies that provide a better preparation of medical experts and better care of the teacher mental health.
\end{abstract}

Key words: Syndrome of Burnout; mental health; teacher health.

\section{SÍNDROME DE BURNOUT: UNA CONFRONTACIÓN ENTRE EL CONOCIMIENTO MÉDICO Y LA REALIDAD DE LAS HISTORIAS CLÍNICAS}

RESUMEN. De la expansión de su práctica, incluyendo, además de la enseñanza, las actividades, la atención para el estudiante y la misión de garantizar el vínculo entre la escuela y la comunidad, el maestro se convirtió en más susceptibles a desarrollar el Síndrome de Burnout, enfermedad profesional cubierto por el Ministerio de Salud desde 1999. Una de las responsabilidades institucionales para su diagnóstico es la experiencia médica. Debido a la importancia del correcto diagnóstico de síndrome de Burnout, este estudio tuvo como objetivo investigar, a través de la aplicación del cuestionario y de la consulta los registros médicos, el conocimiento de los conocimientos médicos de la Sala Médica de la ciudad de João

Doutorado em Saúde Pública pela Fundação Oswaldo Cruz / CPqAM, Brasil (2010). Professor Adjunto da Universidade Federal da Paraíba, Brasil.

II Doutorado em Psicologia Social pela Universidade de Santiago de Compostela, Espanha (2005). Adjunto da Pontifícia Universidade Católica do Rio Grande do Sul, Brasil.

x Doutorado em Engenharia Mecânica pela Universidade de São Paulo, Brasil (1984). Professor pesquisador na Universidade Federal da Paraíba, Brasil

$\Phi$ Doutorado em Ciências Médicas pela Universidade Estadual de Campinas, Brasil (1995). Pesquisador Titular da Fundação Oswaldo Cruz, Brasil 
Pessoa, PB, a partir de la síndrome. Los resultados apuntaron a falta de legislación y del Síndrome de Burnout y la necesidad de políticas públicas que proporcionen una mejor preparación de los expertos médicos y una mejor atención de salud mental del maestro.

Palabras-clave: Síndrome de Burnout; salud mental; salud del profesor.

Nos últimos anos o trabalho docente tem sido objeto de estudo de vários segmentos. De forma geral, os problemas identificados nessa categoria são relacionados à sua própria origem, ao seu desenvolvimento histórico e à valorização social da profissão (Stobäus, Mosquera \& Santos, 2007). Um exemplo desses problemas pode ser encontrado nas reais condições de trabalho do professor, que mobiliza suas capacidades físicas, cognitivas e afetivas, o que não só demanda esforço, mas também gera uma hipersolicitação de suas funções psicofisiológicas. $\mathrm{O}$ professor ampliou sua prática profissional para além da sala de aula, incluindo, além do ensino, atividades de cuidado para com o aluno e a missão de garantir a articulação entre a escola e a comunidade. Esse conjunto de demandas atinge diretamente não só seu desempenho, mas principalmente sua saúde (Gasparini, Barreto \& Assunção, 2005).

A Organização Internacional do Trabalho - OIT identificou a profissão docente como de alto risco, considerando a segunda categoria profissional, em nível mundial, a portar doenças de caráter ocupacional (OIT, 1984). Entre os agravos mais recorrentes relacionados a essa profissão encontram-se os transtornos mentais (Pereira, 2004, Noro \& Kirchhof, 2004). No Brasil, o Ministério da Previdência e Assistência Social (DOU, 1999) apresentou a nova lista de doenças profissionais e relacionadas ao trabalho, a qual contém um conjunto de doze categorias diagnósticas de transtornos mentais. Essas categorias se incluem no que foi chamado de Transtornos Mentais e do Comportamento Relacionados ao Trabalho, os quais podem ser determinados pelos lugares, pelo tempo e pelas ações do trabalho. A décima segunda categoria contemplada é a Síndrome de Burnout, considerada um dos transtornos mentais mais comuns entre os professores e um dos males ocupacionais de caráter psicossocial mais graves na sociedade atual (Salanova \& Llorens, 2008). Trata-se de um fenômeno psicossocial que aparece como uma resposta crônica aos estressores interpessoais vivenciados na situação de trabalho, atingindo profissionais que mantêm uma relação direta e constante com outras pessoas. Esses podem ser médicos, professores, policiais, bombeiros, psicólogos, assistentes sociais, entre outras categorias profissionais (Maslach, Schaufeli \& Leiter, 2001).
A Síndrome de Burnout é um processo que se desenvolve com o passar dos anos e dificilmente é percebida em seus estágios iniciais. Seu desenvolvimento é lento e raramente agudo e seu início é marcado por uma sensação física ou mental de mal-estar indefinida e pela presença de um excessivo e prolongando nível de tensão (Maslach \& Schaufeli, 1993). O Burnout é constituído de três dimensões: exaustão emocional, despersonalização e baixa realização profissional. A exaustão emocional é caracterizada pela falta de energia por certo sentimento de esgotamento de recursos com relação ao trabalho, tendo como maior causa o conflito pessoal nas relações e a sobrecarga de atividades. Está relacionada à carência de recursos emocionais e ao sentimento de inutilidade, tendo como um dos sintomas mais comuns o receio e o temor de voltar ao trabalho no dia seguinte, podendo apresentar manifestações físicas e psíquicas (Santin, 2004). A despersonalização é um estado psíquico no qual prevalece a dissimulação afetiva ou o cinismo e uma tendência a criticar tudo e todos com quem se relaciona no trabalho Por causa do endurecimento afetivo as pessoas acometidas são vistas como desumanas e podendo apresentar sintomas como descomprometimento com os resultados, conduta voltada a si mesmo, alienação, ansiedade, irritabilidade e desmotivação (Codo \& VasquesMenezes, 1999). A baixa realização profissional caracteriza-se pela tendência do trabalhador a se autoavaliar de forma negativa. Ele torna-se insatisfeito com seu desenvolvimento profissional e experimenta um declínio no sentimento de competência e êxito. Há uma diminuição das expectativas pessoais e uma crescente autodepreciação, dando origem a sentimentos de fracasso e baixa autoestima (Codo \& Vasques-Menezes, 1999).

Quando é afetado por essa síndrome o trabalhador se sente exausto, adoece com frequência; passa a ter dores de cabeça, insônia, úlcera; também pode apresentar tensão muscular, fadiga crônica, cefaleias, problemas cardiomusculares, ansiedade e depressão (Salanova \& Llorens, 2008; Gil-Monte, 2005), além de possíveis transtornos psiquiátricos (Gasparini, Barreto \& Assunção, 2006). Sendo esse profissional professor, pode haver mudança radical dos hábitos normais, diminuição da criatividade e do entusiasmo 
com o trabalho, dificuldade de concentração, perda do autorrespeito e do autocontrole na sala de aula e reações exageradas na tentativa de diminuir o estresse, além do desenvolvimento, a longo prazo, de doenças como úlcera, hipertensão, depressão e alcoolismo (Yong \& Yue, 2007). Devido à alta incidência entre os professores do mundo inteiro, a Síndrome de Burnout está sendo considerada uma epidemia no campo educacional (Gil-Monte, 2005), devendo ser tratada como uma importante questão de saúde pública (Salanova \& Llorens, 2008).

Considerada uma doença relacionada ao trabalho, a Síndrome de Burnout tem como principal instrumento de diagnóstico institucional o médico perito. Geralmente o trabalhador procura afastamento de suas atividades em decorrência de queixas relacionadas a sintomas físicos que o impossibilitam de exercer sua função e são causas de sofrimento físico e/ou psíquico. Cabe ao médico perito identificar que tipo de agravo está relacionado às queixas e o tempo de afastamento do trabalho suficiente para a recuperação, assim como a relação entre a doença e a atividade laboral do trabalhador.

O médico perito é um profissional que deve ter conhecimento necessário das leis, normas, portarias, profissiografia, além de ter capacidade de avaliar a associação das patologias à atividade do trabalhador e a todos os fatores associados à sua capacidade laborativa. Soma-se a isso a necessidade de uma sólida formação clínica, amplo domínio da legislação, disciplina legal e administrativa, além de atributos de caráter e personalidade (integridade, independência, equilíbrio) (Cabral, 1997).

É de relevante importância o correto diagnóstico, pois a não caracterização do papel do trabalho como agravante ou desencadeante de distúrbios psíquicos ocasiona prejuízos não só à qualidade e à eficácia do tratamento mas também aos direitos legais do trabalhador, que deixa de usufruir de benefícios previdenciários aos quais eventualmente tenha direito (Glina, Rocha, Batista \& Mendonça, 2001). Acrescenta-se a essa questão o fato de que, na maioria das vezes, as doenças ocupacionais tornam-se incuráveis, devido ao longo tempo para a realização do seu correto diagnóstico. Em alguns países, como a Suécia e a Holanda, um diagnóstico formal da SB abre a possibilidade de afastamento, tratamento e compensação financeira (Schaufeli, Leiter \& Maslach, 2009). Após a introdução de seu diagnóstico na Suécia em 1997, a SB tornou-se um dos cinco diagnósticos mais comuns, com evidente tendência de aumento, principalmente no setor público (Friberg, 2006).
Considerando os atributos necessários à perícia médica e a importância do diagnóstico da Síndrome de Burnout na categoria docente, esse estudo buscou, além de identificar o conhecimento a respeito da Síndrome de Burnout em um serviço público de perícia médica, confrontar tal conhecimento com os diagnósticos realizados pelos peritos.

\section{MÉTODO}

\section{Participantes}

Participaram do estudo doze profissionais (seis homens e seis mulheres) em atividade dos catorze que compõem o quadro de Perícia Médica da Junta Médica Municipal da Cidade de João Pessoa, PB. A idade média era de 51,6 anos ( $\mathrm{DP}=8,2)$, e tempo de serviço médio, de 18,5 anos $(\mathrm{DP}=9)$.

\section{Instrumentos}

Foi utilizado um questionário autoaplicável, elaborado pelos pesquisadores do estudo. $\mathrm{O}$ questionário foi composto por questões objetivas que investigaram os seguintes pontos: se conheciam a lei da Previdência que inclui a Síndrome de Burnout; se foram submetidos a treinamento para diagnosticar o Burnout; se já haviam tido diagnóstico de Bournout ou tinham conhecimento de algum colega que o tivesse tido; se algum professor já foi afastado do trabalho em decorrência do Burnout.

A fim de confirmar as informações dos peritos de que houve algum diagnóstico e/ou afastamento de professores do trabalho em decorrência da Síndrome de Burnout entre os anos de 1999 (ano em que foi publicada a portaria que incluiu essa síndrome como doença do trabalho) e 2006, foi realizado um estudo exploratório nas 1.014 fichas médicas individuais de todos os professores da rede municipal de ensino que se encontravam lotados na Secretaria de Educação da Prefeitura e haviam passado pela Junta Médica Municipal nesse período.

\section{Procedimentos}

Primeiramente foi realizado contato com os participantes a fim de expor os objetivos do estudo. Foi esclarecido que se tratava de uma pesquisa sem quaisquer efeitos avaliativos individuais e/ou institucionais e que as respostas seriam anônimas e confidenciais. A aplicação foi feita de forma individual, no final do expediente de trabalho, e recolhido após seu preenchimento. A média de tempo de resposta foi de 30 minutos. 
O banco de dados foi construído após autorização da Secretaria de Administração do Município de João Pessoa, sendo realizada, a partir daí, a digitação dos dados individuais das fichas médicas, os quais posteriormente foram analisados por meio do SPSS 15.0 (Statistical Package for the Social Sciences). Foi feita análise descritiva das respostas médicas e da prevalência de transtornos mentais que afastaram o professor do trabalho de 1999 a 2006.

O projeto de pesquisa foi aprovado pelo Comitê de Ética em Pesquisa do Centro de Ciências da Saúde da Universidade Federal da Paraíba. Todos os participantes do estudo receberam e assinaram o Termo de Consentimento Livre e Esclarecido (Certidão do Comitê de Ética em Pesquisa do Centro de Ciências da Saúde da Universidade Federal da Paraíba - CEP/CCS/UFPB - número 0116).

\section{RESULTADOS}

As respostas do questionário aplicado aos médicos peritos mostraram os seguintes resultados: nenhum perito recebeu informação das instâncias superiores a respeito da atualização da legislação previdenciária; $75 \%$ não conhecem a portaria que inclui a Síndrome de Burnout como doença do trabalho; nenhum perito recebeu treinamento para lidar com a Síndrome de Burnout em sua prática profissional; $75 \%$ não têm conhecimento da Síndrome de Burnout; $83,3 \%$ não fizeram nenhum diagnóstico da Síndrome de Burnout; 91,7\% não têm conhecimento de algum colega que tenha feito diagnóstico da Síndrome de Burnout; 91,7\% não afastaram nenhum professor do trabalho em decorrência da Síndrome de Burnout (tabela 1).

Tabela 1 - Questionário aplicado aos Médicos Peritos

\begin{tabular}{|c|c|c|c|c|c|c|}
\hline \multirow[t]{2}{*}{ Questões } & \multicolumn{2}{|c|}{ SIM } & \multicolumn{2}{|c|}{ NÃO } & \multicolumn{2}{|c|}{ TOTAL } \\
\hline & $\mathbf{F}$ & $\%$ & $\mathbf{F}$ & $\%$ & $\mathbf{F}$ & $\%$ \\
\hline $\begin{array}{l}\text { Você recebe alguma informação das instâncias superiores a respeito da atualização da Legislação } \\
\text { Previdenciária? }\end{array}$ & 0 & 0,0 & 12 & 100 & 12 & 100 \\
\hline $\begin{array}{l}\text { Você conhece a portaria } n^{\circ} .89 \text { do Ministério da Previdência e Assistência Social (DOU } \\
\text { 12.05.1999) que inclui a Síndrome de Burnout no campo das Doenças do Trabalho? }\end{array}$ & 3 & 25,0 & 9 & 75,0 & 12 & 100 \\
\hline $\begin{array}{l}\text { Você já recebeu algum treinamento para lidar com a Síndrome de Burnout em sua prática } \\
\text { profissional? }\end{array}$ & 0 & 0,0 & 12 & 100 & 12 & 100 \\
\hline Você tem conhecimento da Síndrome de Burnout? & 3 & 25,0 & 9 & 75,0 & 12 & 100 \\
\hline Você já fez algum diagnóstico da Síndrome de Burnout? & 2 & 16,7 & 10 & 83,3 & 12 & 100 \\
\hline Você tem conhecimento de algum colega que tenha feito diagnóstico da Síndrome de Burnout? & 1 & 8,3 & 11 & 91,7 & 12 & 100 \\
\hline Você já afastou algum professor do trabalho em decorrência da Síndrome de Burnout? & 1 & 8,3 & 11 & 91,7 & 12 & 100 \\
\hline
\end{tabular}

Fonte: Questionário aplicado à Perícia Médica da Junta Médica Municipal da cidade de João Pessoa, PB.

A partir da avaliação das fichas médicas individuais dos professores (tabela 2) constatou-se que os transtornos mentais e comportamentais foram responsáveis por $13,46 \%$ dos afastamentos. Entre os transtornos mentais o diagnóstico de depressão ocupa um lugar de destaque, sendo responsável pela metade dos afastamentos (41\% por episódios depressivos e
$8,3 \%$ por transtornos depressivos recorrentes). Se considerarmos também que outros transtornos ansiosos $(14,8 \%)$, reações ao estresse grave $e$ transtorno de adaptação $(14,8)$ transtornos fóbicoansiosos (4,2\%), transtorno de humor $(2,6 \%) \mathrm{e}$ transtorno afetivo bipolar $(1,6 \%)$ possuem sintomas de depressão, o percentual eleva-se para $87,3 \%$.

Tabela 2 - Transtornos mentais que afastaram o professor do trabalho de 1999 a 2006

\begin{tabular}{lc}
\hline Transtornos Mentais (CID 10) & Frequência (\%) \\
\hline Episódios depressivos (F32) & $227(41,0)$ \\
Outros transtornos ansiosos (F41) & $82(14,8)$ \\
Reações ao estresse grave e transtorno de adaptação (F43) & $82(14,8)$ \\
Transtorno depressivo recorrente (F33) & $46(8,3)$ \\
Transtornos fóbico-ansiosos (F40) & $23(4,2)$ \\
Transtornos psicóticos agudos e transitórios (F23) & $16(2,9)$ \\
Transtorno de humor (F38) & $14(2,6)$ \\
Transtornos psicóticos não orgânicos (F28) & $12(2,2)$ \\
Transtorno obsessivo-compulsivo (F42) & $9(1,6)$ \\
Transtorno afetivo bipolar (F31) & $9(1,6)$ \\
Outros transtornos mentais que tiveram menos de 1,0\% & $33(6,0)$ \\
Total & $553(100,0)$ \\
\hline
\end{tabular}

Fonte: Junta Médica Municipal da cidade de João Pessoa, PB 


\section{DISCUSSÃO}

Por esses resultados se pode concluir que Perícia Médica desconhece a Síndrome de Burnout. A portaria que incluiu essa síndrome como doença do trabalho (Ministério da Saúde, 1999) foi publicada no ano de 1999 e ainda é desconhecida de $75 \%$ dos médicos da Junta. Esse desconhecimento justifica a impossibilidade de diagnóstico (83,3\% afirmam nunca ter feito um diagnóstico de Burnout) e o fato de a maioria não conhecer nenhum colega em que também tenha sido diagnosticada a síndrome. Ademais, apesar de $16,7 \%$ terem afirmado que já tiveram esse diagnóstico e de 8,3\% terem afirmado que já afastaram professor do trabalho em consequência da Síndrome de Burnout, não foi identificado em nenhuma ficha médica entre os anos de 1999 e 2006 diagnóstico e/ou afastamento do trabalho em decorrência dessa síndrome.

Desde o início dos estudos sobre Burnout diversos autores (Bakker, Schaufeli, Demerouti, Janssen, Van Der Hulst \& Brouwer, 2000; Farber, 1991; Gomez de Cadiz, San Juan, Riviero, Herce \& Acucarro, 1997; Maslach \& Jackson, 1981; Toker, Shirom, Shapira, Berliner \& Melamed, 2005) têm alertado sobre a necessidade de realizar um adequado diagnóstico diferencial com relação à depressão, tendo em vista que alguns sintomas da síndrome são semelhantes. Maslach e Jackson (1981) pontuam que, embora sejam construtos relacionados, não são sinônimos. Os quadros que incluem sintomas depressivos apresentam alterações no apetite, no peso ou no sono, diminuição da energia, sentimentos de desvalia ou culpa, dificuldades em pensar, concentrar-se ou tomar decisões, pensamentos recorrentes sobre morte ou ideação suicida e podem ser um fator de confusão no diagnóstico de Burnout.

Destaca-se que nenhum dos diagnósticos listados se refere a transtornos mentais e do comportamento relacionados ao trabalho. Neste sentido, pode-se pensar em percentuais superiores aos obtidos no presente estudo, uma vez que, de acordo com Glina, Rocha, Batista e Mendonça (2001), embora apresentem alta incidência entre a população trabalhadora, os distúrbios psíquicos relacionados ao trabalho frequentemente deixam de ser reconhecidos como tais no momento da avaliação clínica. Contribuem para isto, entre outros motivos, as próprias características dos distúrbios psíquicos, regularmente mascarados por sintomas físicos, bem como a complexidade inerente à tarefa de definir claramente a associação entre tais distúrbios e o trabalho desenvolvido pelo paciente.
Todos os anos são registrados cerca de $30 \mathrm{mil}$ casos de doenças do trabalho no Brasil. Embora significativo, o número representa apenas $5 \%$ dos casos oficiais verificados nos Estados Unidos, onde essa cifra chega a 600 mil. Essa diferença não se deve à existência de melhores condições de trabalho no Brasil, mas revela o retrato de um crônico problema brasileiro: a precariedade das estatísticas ou a inexistência delas. Esses números são tão baixos no Brasil porque as doenças do trabalho ou não são diagnosticadas corretamente ou, quando o são, não são registradas como tal (Almeida, citado por Silveira, 2000). Para o autor, o despreparo dos profissionais da medicina para elaborar diagnósticos de doenças profissionais é uma das razões para essas distorções estatísticas. Dias, Silveira, Chiavegatto \& Resende (2006) apontam dificuldades no ensino sistemático das relações saúde-trabalho-doença nos cursos de medicina do Brasil. A pouca importância dada a essa temática na educação médica ocorre, entre outros fatores, pela visão distorcida de que se trata de uma disciplina leve e que pode ser aprendida na vida profissional. Assim, ainda prepondera o paradigma clássico da medicina, que procura entender os processos mórbidos ocupacionais através de uma abordagem organicista, além do as demandas físicas serem mais fáceis de definir e medir do que as mentais (Borsoi, 2007; Lima, Assunção \& Francisco, 2002).

A constatação, neste estudo, de que não houve nenhum diagnóstico da Síndrome de Burnout entre os professores da rede municipal de ensino entre os anos de 1999 (ano em que a legislação incluiu essa síndrome como doença do trabalho) e 2006 aponta, não para a inexistência dessa síndrome na categoria, mas para a falta de conhecimento e de condições de identificar essa patologia por parte dos médicos peritos. Estudos realizados no Brasil e em outros países, que utilizaram um instrumento específico, o MBI- Maslach Burnout Inventory de Maslach \& Jackson (1981) para avaliar a Síndrome de Burnout, evidenciaram na categoria docente índices de incidência preocupantes (Batista et al., 2010; Carlotto \& Palazzo, 2006; Carvalho, 2003; Dorman, 2003a, 2003b; Maslach, 2003).

O desconhecimento da legislação por parte da maioria dos médicos peritos de uma instituição que tem como principal objetivo avaliar as condições de saúde do trabalhador (Perícia Médica) e o distanciamento das instâncias superiores responsáveis pela capacitação e fiscalização desses profissionais corroboram a ideia de abandono e descaso com relação ao trabalhador, nesse caso, o trabalhador docente. Também revelam a necessidade de esses 
profissionais investirem e aprofundarem estudos sobre estas questões, dando mais destaque aos seus objetivos de trabalho, que é proporcionar uma melhor qualidade de vida ao trabalhador (Carlotto, 2001). O Decreto 3.048/99 de 6 de maio de 1999 representa um avanço, mas traz um desafio: reconhecer, diagnosticar e efetuar o nexo causal dos transtornos mentais com o trabalho.

A necessidade de estabelecer o nexo causal entre o adoecimento e a situação de trabalho exige do médico perito uma postura diferenciada que valorize o processo específico de cada indivíduo, considerando sua história de vida e de trabalho, ou seja, o ambiente, a organização e a percepção da influência do trabalho na construção da doença (Glina et al., 2001). Segundo Borsoi (2007), ainda hoje, grande parte dos médicos não costuma se preocupar em saber como seus pacientes trabalham para viver. É comum manter-se um suposto distanciamento entre trabalho e saúde mental, como se o primeiro não pudesse dizer nada sobre o segundo, ou como se determinados aspectos objetivos e subjetivos do trabalho não pudessem provocar adoecimento.

Assim, o primeiro ponto é reconhecer que o contexto de trabalho é complexo e multifacetado e possui estreita relação com o adoecimento ocupacional (Chopra, 2009). No caso da Síndrome de Burnout esse entendimento é fundamental, pois se trata de uma doença ocupacional relacionada à inadequada organização do trabalho. O médico precisa atender o paciente de forma contextualizada, realizando uma escuta diferenciada e uma investigação que vá além da queixa principal apresentada, geralmente desvinculada do contexto de trabalho. Segundo Carlotto (2001), não raras vezes, em situação de conflito, o trabalhador esconde e sonega informações que possam contribuir para um adequado diagnóstico e tratamento. $\mathrm{Na}$ perspectiva do trabalhador, pensar e referir o trabalho como elemento de adoecimento, estabelecendo cognitivamente esse nexo causal, ocasiona ansiedade e temores, pois o levaria à tomada de decisões conflitivas, uma delas a possível saída do trabalho.

Considerando todas essas dificuldades já apontadas, Glina et al. (2001) pontuam a necessidade da capacitação de profissionais dos serviços de saúde para que considerem a importância da situação de trabalho como um dos determinantes no processo saúde/doença. Esses autores também sugerem a reestruturação dos sistemas de informações em saúde, envolvendo um sistema de vigilância epidemiológica com notificação dos casos com suspeita de relação com o trabalho, independentemente da caracterização por parte da Previdência Social; incluindo o desenvolvimento de ações interinstitucionais e multidisciplinares em Saúde Mental e Trabalho.

\section{CONCLUSÃO}

A realidade investigada aponta certo distanciamento entre o que efetivamente é avaliado com relação à saúde mental do professor e a condição apresentada pela instituição responsável por essa avaliação. Pode-se questionar até que ponto os resultados observados na Junta Médica da cidade de João Pessoa se repetem em outras instituições, indagando se a Síndrome de Burnout está sendo incluída na avaliação da saúde mental do professor ou se a própria categoria docente tem conhecimento do que pode estar por trás dos sintomas clássicos de estresse que apresentam. Essas questões sugerem a continuidade desse estudo e a necessidade de novos espaços de investigação, pois é preciso que haja medidas de intervenção por parte das políticas públicas no sentido de valorizar o professor e cobrar maior eficiência no que se refere à avaliação de sua saúde. Essas medidas devem considerar uma realidade em que a saúde mental da categoria docente está comprometida, atingindo, consequentemente, seu desempenho no trabalho, suas relações pessoais e a qualidade de sua educação.

\section{REFERÊNCIAS}

Bakker, A. B., Schaufeli, W. B., Demerouti, E., Janssen, P. P. M., Van Der Hulst, R., \& Brouwer, J. (2000). Using equity theory to examine the difference between burnout and depression. Anxiety, Stress, and Coping, 13, 247-268.

Batista, J. B. V., Carlotto, M. S., Coutinho, A. S., Augusto, L. G. S. (2010). Prevalência da Síndrome de Burnout e fatores sociodemográficos e laborais em professores de escolas municipais da cidade de João Pessoa, PB. Revista Brasileira de Epidemiologia, 13 (3), 502-512.

Borsoi, I. C. F. (2007). Da relação entre trabalho e saúde à relação entre trabalho e saúde mental. Psicologia \& Sociedade, 19(1) (Edição Especial), 103-111.

Cabral, E. C. F. (1997). Manual de perícia médica. Brasília: Ministério da Saúde.

Carlotto M. S., \& Palazzo L. S. (2006). Síndrome de Burnout e fatores associados: um estudo epidemiológico com professores. Cadernos de Saúde Pública, 22(5),1017-1026.

Carlotto, M. S. (2001). O trabalho humano e a saúde mental. Aletheia, 14, 85-98.

Carvalho, F. A. (2003). O mal-estar docente: das chamas devastadoras (Burnout) às flamas da esperança-ação (resiliência). Dissertação de Mestrado. Pontifícia Universidade Católica de São Paulo, São Paulo, SP. 
Chopra, P. (2009) Mental health and the workplace: issues for developing countries. International Journal of Mental Health Systems, 3(4),1-9.

Codo W. \& Vasques-Menezes (1999). O que é burnout? In W. Codo (Org.), Educação: Carinho e Trabalho. (pp. 237-260). Petrópolis: Vozes.

Decreto n. 3048, de 6 de maio de 1999. (1999, 6 de maio) Aprova o Regulamento da Previdência Social, e dá outras providências. Brasilia: Ministério da Saúde. Recuperado em 3 de agosto, de 2010

de http://www010.dataprev.gov.br/sislex/paginas/23/1999/3048.htm.

Dias, C. E., Silveira, A. M., Chiavegatto, C. V., \& Resende, N. P. (2006). O ensino das relações trabalho-saúde-doença nas escolas médica: percepção dos alunos e proposta de aperfeiçoamento na UFMG. Revista Brasileira de Educação Médica, 30(1), 1-7.

Dorman, J. (2003a). Testing a model of teacher Burnout. Australian Journal of Educational \& Developmental Psychology, 3, 35-47.

Dorman, J. (2003b). Relationship between school a classroom environment and teacher Burnout: a LISREL analysis. Social Psychology of Education, 6(2),107-127.

Farber B. A. (1991). Crisis in education. Stress ans burnout in American teacher. San Francisco: Jossey-Bass.

Friberg T. (2006) Diagnosing burn-out: An anthropological study os social concept in Sweden. (Unpublished master's thesis). Lund University, Lund, SW.

Gasparini, S. M., Barreto, S. M., \& Assunção, A (2005). O professor, as condições de trabalho e os efeitos sobre sua saúde. Educação $e$ Pesquisa, 31(2), 189-199.

Gasparini, S. M., Barreto, S. M., \& Assunção, A. A. (2006). Prevalência de transtornos mentais comuns entre professores da rede municipal de ensino de Belo Horizonte. Cadernos de Saúde Pública, 22(12), 2.679-2.691.

Gil-Monte P. R. (2005) El syndrome de quemarse por el trabajo ("burnout"). Una enfermedad laboral en la sociedad del bienestar. Madrid: Pirámide.

Glina, D. M. R., Rocha, L. E., Batista, M. L., \& Mendonça, M. G. V. (2001). Saúde mental e trabalho: uma reflexão sobre o nexo com o trabalho e o diagnóstico, com base na prática. Cadernos de Saúde Pública, 17(3),607-616.

Gomez de Cadiz, B. T., San Juan, C., Riviero, A. M., Herce, C., \& Achucarro, C. (1997). Burnout professional: un problema nuevo? Reflexões sobre el concepto y su evaluación. Revista de Psicologia del Trabajo y de las Organizaciones, 13(1), 23-50.

Lima, M. E. A., Assunção, A. A., \& Francisco, J. M. S. D. (2002). Aprisionados pelos ponteiros de um relógio: o caso de um transtorno mental desencadeado pelo trabalho. In M. G. Jacques, \& W. Codo (Orgs.), Saúde Mental \& Trabalho-Leituras (pp.5472). Petrópolis: Vozes.

Maslach C. (2003). Burnout: the cost of caring. Englewood Cliffs, N. J.: Pretince-Hall.
Maslach, C., \& Jackson, S. E. (1981). The measurement of experienced burnout. Journal of Ocuppational Behavior, 2, 99113.

Maslach, C., \& Schaufeli W. B. (1993). Historical and conceptual development of burnout. In W. B. Schaufeli, C. Maslach, \& T. Marek (Orgs.), Professional Burnout: recent developments in theory and research (pp.1-16). New York: Taylor \& Francis.

Maslach, C., Schaufeli, W.B., \& Leiter M.P. (2001). Job burnout. Annual Review Psychology, 52, 397-422.

Noro, C. P., \& Kirchhof, A. L. C. (2004). Prevalência dos transtornos mentais em trabalhadores de instituição federal de ensino superior. Saúde, 30(1-2),104.

Organização Internacional do Trabalho (1984). A condição dos professores: recomendação Internacional de 1966, um instrumento para a melhoria da condição dos professores. Genebra: OIT/ Unesco.

Pereira, A. P. T (2004). Os profissionais de educação e as doenças que os afastam de sala de aula. Monografia de especialização, Centro de Tecnologia, Universidade Federal da Paraíba, João Pessoa, PB, Brasil.

Salanova, M.Y., \& Llorens, S. (2008). Estado actual y retos futuros en el estudio del burnout. Papeles del Psicólogo, 29(1),59-67.

Santin, J. (2004). Síndrome do esgotamento profissional-Revisão Bibliográfica. Revista Movimento, 10(1),183-209.

Schaufeli, B. W., Leiter, M. P., \& Maslach, C. (2009). Burnout: 35 years of research and practice. Career Development International,14(3), 204-220.

Silveira, E. (2000). Comissão cria nova Lista de Doenças Profissionais, que passa a incluir as lesões por esforço repetitivo e os distúrbios psicológicos e mentais. Jornal de Medicina Ocupacional [on line]. Recuperado em 22 dezembro, de 2000, de www.unesp.br/.

Stobäus, C., Mosquera, J., \& Santos, B. (2007). Grupo de pesquisa mal-estar e bem-estar na docência. Revista Educação, 30(n. especial), 259-272.

Toker, S., Shirom, A., Shapira, I., Berliner, S., \& Melamed, S. (2005). The association between burnout, depression, anxiety, and inflammation biomarkers: c-reactive protein and fibrinogen in men and women. Journal of Occupational Health Psychology, 10(4), 344-362

Yong, Z., \& Yue, Y. (2007). Causes for burnout among secondary and elementary school teachers and preventive strategies. Chinese Education and Society, 40(5),78-85.

Recebido em 01/09/2010 Aceito em 03/11/2011

Endereço para correspondência:

Jaqueline Brito Vidal Batista. Rua Áurea, 67 - Ap 201, Cabo Branco, CEP 58045-360, João Pessoa-PB, Brasil.E-mail: jaquebvb@gmail.com. 